\title{
Diffusion of innovation in mental health policy adoption: what should we ask about the quality of policy and the role of stakeholders in this process?

\author{
Comment on "Cross-national diffusion of mental health policy"
}

\section{Lucy Lee ${ }^{*}$}

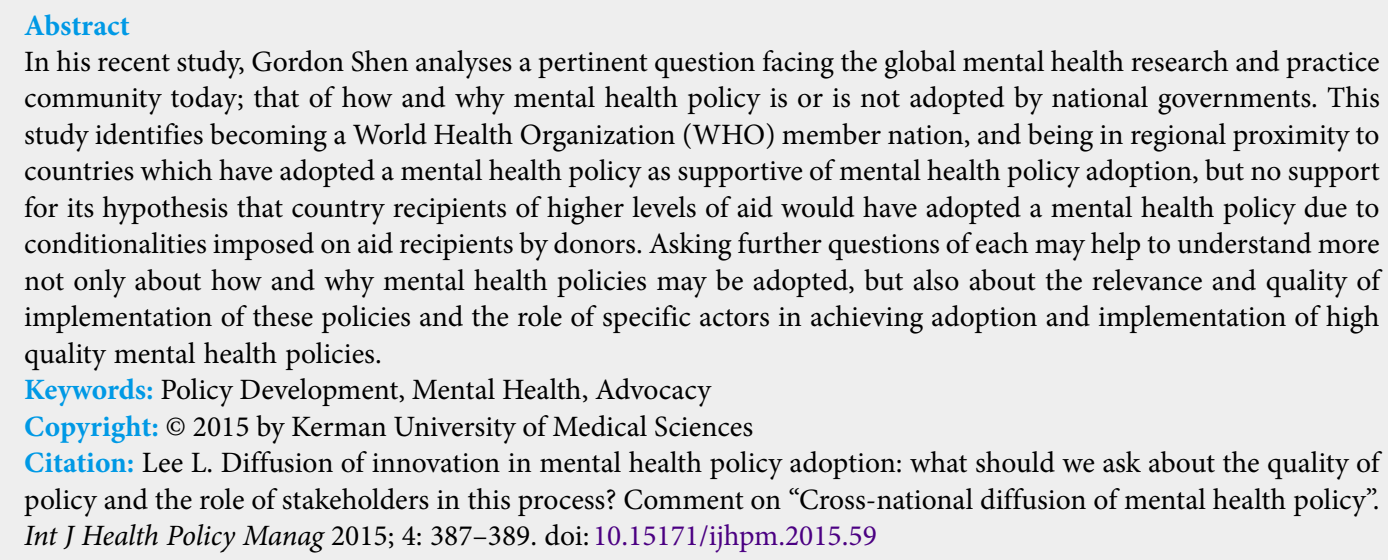
community today; that of how and why mental health policy is or is not adopted by national governments. This study identifies becoming a World Health Organization (WHO) member nation, and being in regional proximity to countries which have adopted a mental health policy as supportive of mental health policy adoption, but no support for its hypothesis that country recipients of higher levels of aid would have adopted a mental health policy due to conditionalities imposed on aid recipients by donors. Asking further questions of each may help to understand more not only about how and why mental health policies may be adopted, but also about the relevance and quality of implementation of these policies and the role of specific actors in achieving adoption and implementation of high quality mental health policies.

Keywords: Policy Development, Mental Health, Advocacy

Copyright: @ 2015 by Kerman University of Medical Sciences

Citation: Lee L. Diffusion of innovation in mental health policy adoption: what should we ask about the quality of policy and the role of stakeholders in this process? Comment on "Cross-national diffusion of mental health policy". Int J Health Policy Manag 2015; 4: 387-389. doi: 10.15171/ijhpm.2015.59

\section{Article History:}

Received: 23 January 2015 Accepted: 6 March 2015 ePublished: 10 March 2015

*Correspondence to:
Lucy Lee
Email: lucy.lee@lshtm.ac.uk
$\mathrm{I}$ $\mathrm{n}$ his recent study, Gordon Shen applies a novel methodological framework to analyse a pertinent question facing the global mental health research and practice community today; that of how and why mental health policy is or is not adopted by national governments (1). In doing so, he begins a dialogue not only about deepening our understanding of the processes that underlie the adoption of mental health policy within and - crucially - between countries, but also about the role of influencing bodies in achieving this.

This study's focus on diffusion of innovation is important, as it is widely acknowledged that adequate policy development and implementation is limited in mental health $(2,3)$. Understanding the routes by which policy development is positively influenced by actions in other jurisdictions and by specific actors may help to strengthen approaches to improve policy adoption, particularly in areas where there is low investment in this (4). However, I would argue that as, if not more important than understanding the influences on the adoption of mental health policy by specific countries, is understanding what affects the relevance of that policy in relation to need, and the efforts made to finance, implement and measure the success of that policy and its related implementation plan.

Shen's study provides an important framework and key observations for analysing in depth patterns of significance in the adoption of mental health policies over time through the application of quantitative methods, analyzing relevant World Health Organization (WHO) and Official Development
Assistance (ODA) and Development Assistance for Health (DAH) datasets. The results of this study identify two factors supporting policy adoption; becoming a WHO member nation, and being in regional proximity to countries which have adopted a mental health policy (a so-called contagion effect). In contrast, this study found no support for its hypothesis that country recipients of higher levels of general development aid would have adopted a mental health policy due to conditionalities imposed on aid recipients by donors. To build on and deepen these findings then, we might ask further questions of each in turn - in order to understand more not only about the adoption of mental health policies in this context, but also about the relevance and quality of implementation of these policies and the role of specific actors in achieving these - and to identify appropriate methods to answer these questions.

The influence of membership in the World Health Organization (WHO)

The WHO does indeed play an important role in building member states' capacities to adopt and implement mental health policy. A recent development (coming after the period of analysis of this study) which has the potential to contribute significantly to the WHO's ability to provide support to nations in adopting appropriate and pertinent mental health policies, was the ratification of the WHO Mental Health Action Plan 2013-20 by 194 Member States in May 2013. While global in scope, drawing from regional WHO action plans and strategies for mental health, and aligning with other 
global WHO action plans, the mental health action plan is designed to provide guidance for national mental health action plans (5). Of the plan's four objectives, the first includes a global target that $80 \%$ of countries will have developed or updated their policies or plans for mental health in line with international and regional human rights instruments by the year 2020.

The questions that arise from this development speak to the role of the WHO, Member States and international organisations in achieving these targets and to how and whether a global framework such as the Mental Health Action Plan can improve the adoption and implementation of mental health policy at a national level. Of course, this is plausible; it has been suggested that acceleration of policy development in the early 2000s in Sub-Saharan Africa may be linked to recommendations made in the World Health Report 2001, but while we are aware of an increase in the existence of mental health policies in this context, we know far less about the relevance and quality of implementation of these policies (6). Issues raised repeatedly in relation to developing effective mental health policy include assessing the current situation, involving key stakeholders, consulting the evidence base, aligning with other priority health policies and nonhealth policies (e.g. social welfare), setting goals and targets, strategic plans and implementation frameworks and regularly reviewing progress and revising strategies as necessary. Of course fundamental to all of this is adequately resourcing a policy $(7,8)$.

Monitoring the implementation of the Mental Health Action Plan within specific countries and regions [as efforts to create regional action plans are initiated (9)] provides an opportunity to assess the factors which contribute to its successfully influencing policy adoption and implementation at various levels, and could also illuminate much about the factors influencing policy relevance and quality of implementation. Case studies which determine the extent to which targets are achieved within specific jurisdictions and the role of specific actors could be invaluable in understanding better these factors (10). Although somewhat time and resource intensive, a small number of these studies triangulated with quantitative analysis of action plan implementation monitoring data may provide a rich additional resource for better understanding and therefore acting upon factors that facilitate or impede policy adoption and implementation at the national or regional level.

\section{Diffusion of innovation regionally and between countries} While Shen focuses on the independent variable of 'contagion effect within regions', in determining the extent to which diffusion of innovation in policy adoption takes place across jurisdictions, he also touches upon the potential influence of regional and global 'learning and emulation mechanisms' on policy adoption. Here lies an area for further research. The advocacy potential of initiatives such as the Grand Challenges in Global Mental Health Initiatives, the Mental Health Innovation Network (MHIN), and the Gulbenkian Platform for Global Mental Health to accelerate efforts in getting mental health onto policy agendas should not be overlooked. How they might coordinate and evaluate their work in order to maximize their impact in influencing policy is a fundamental question to ask, and one which could provide resources for the global health community for improved policy influence and opportunities to scale up cost-effective innovations in mental health.

These initiatives aim to focus attention and action on improving investment in mental health research and service development globally. In this regard, they can learn from other successful global health movements, as suggested by the Overseas Development Institute in a recent context analysis of global mental health policy (11). Take a sector such as HIV/AIDS - an area of health which now attracts more than a third of global donor health financing (up from around seven percent a decade ago) - which has been influenced by a powerful, unified movement with a clear policy ask. The campaign was sustained and sophisticated, using powerful methods to frame the issue, develop a targeted communications strategy and ensure their approach was selective and timely.

MHIN (http://www.mhinnovation.net), a partnership between the London School of Hygiene \& Tropical Medicine and the WHO funded by Canadian donor Grand Challenges Canada, aims to translate mental health research into policy and increase international prioritization of mental health, and can learn from the successes of the HIV/AIDS movement to achieve this. Global movements like MHIN are positioned to provide support to national and regional efforts to influence policy through developing strategies and resources to guide these activities. The success of these approaches could benefit from research undertaken by initiatives like MHIN such as case studies mapping the political environment in priority countries (12) which assess political priority for improving mental health, determine the factors which affect this and identify barriers and successes experienced by advocates in increasing the prioritization of mental health. The results of these case studies could then be used to develop plans for generating political commitment within these countries and provide guidance on suitable approaches to be used in other countries.

Movements like MHIN can play a convening role in creating opportunities for knowledge exchange and fostering links between the research and decision-making community. Being positioned to be able to call upon a global network of innovators and a bank of case studies of cost-effective, evidence-based approaches to scaling up mental healthcare to support the development of key messages can add support to advocacy efforts. MHIN has developed a knowledge exchange strategy (13) to coordinate efforts to provide generators of knowledge with the tools and skills to communicate impact effectively to decision-makers, and equally to enable decision-makers to access and use evidence to make appropriate decisions that lead to improved mental health services.

\section{Aid and policy adoption}

Shen acknowledges two important points in assessing the influence of aid on policy adoption. The first is that understanding the mechanisms of aid flows and the conditionalities assigned to them is complex. The second is that it is very difficult to tease out the specific mental health activities funded through ODA, as coding of this information 
is not possible. Although Shen's study finds no support for the prediction that levels of aid may influence policy adoption, determining why this is the case may be supported by conducting an analysis of international donor commitment to the implementation of interventions that aim to improve the mental health of populations. This would help to answer several questions, first whether and how international aid agencies provide support for, or indeed impose specific conditions on recipient countries, and following from this, whether and how aid influences the adoption of mental health policies. Having an accurate picture of the current donor landscape and priorities in funding to mental health provides an advocacy tool, both where it can be demonstrated that funding makes a positive impact (and in this regard donors have a responsibilities to assess the collective impact of their funding decisions), and in highlighting the gaps, needs and priorities for additional and targeted funding to mental health.

Considering the potential of the Mental Health Action Plan to support policy adoption and implementation globally, advocacy efforts may also be made to influence institutional donors to fund activities that support the implementation of this plan. The UK All Party Parliamentary Groups on mental health and global health published a report in November 2014 calling for increased funding to mental health in Lowand Middle-Income Countries (LMICs) (14). It aimed these recommendations at four UK stakeholders, including the Department for International Development (DfID). This report represents a powerful advocacy effort, raising the levels and focus of funding to mental health programmes in LMICs by the UK government into the spotlight. It promotes approaches for improved engagement by the UK government in tailoring its overseas development assistance to develop a roadmap to providing assistance to countries to achieve the WHO Mental Health Action Plan objectives.

With these recommendations now under consideration by the UK government, this process may provide a template for continued advocacy efforts to ensure that at a national level, agencies responsible for providing assistance to governments in LMICs ensure they include mental health into health system strengthening packages of support.

\section{Conclusion}

Shen highlights three important factors in determining whether mental health policies are adopted by Member States. There is a role across each of the areas explored in this study for advocacy and research activities to aid progress in supporting countries to adopt and implement high quality, relevant, and adequately-resourced mental health policies. Creating the conditions for this to be achieved requires deeper analysis of the barriers and enablers to adoption of policy by individual countries, and how countries and regions may be more powerfully influenced to do this. It also requires the sustained engagement of WHO and other international bodies to implement frameworks to support this, and the coordination of global movements to initiate and support policy influence activities. Crucially, scrutinizing the implementation of national mental health action plans and policies through the triangulation of qualitative studies and quantitative analysis is much needed. Future research into mental health policy adoption should prioritise a mixed methods approach that utilizes routine, comparable monitoring data such as that generated through national mental health action plans and in-depth, qualitative studies.

Ethical issues

Not applicable.

Competing interests

Author declares that she has no competing interests.

Author's contribution

$\mathrm{LL}$ is the single author of the manuscript.

References

1. Shen GC. Cross-national diffusion of mental health policy. Int J Health Policy Manag 2014; 3: 269-82. doi: 10.15171/ ijhpm.2014.96

2. Knapp M, Funk M, Curran C, Prince M, Grigg M, McDaid D. Economic barriers to better mental health practice and policy. Health Policy Plan 2006; 21: 157-70. doi: 10.1093/ heapol/czl003

3. Saxena S, Thornicroft G, Knapp M, Whiteford H. Resources for mental health: scarcity, inequity, and inefficiency. Lancet 2007; 370: 878-89. doi: 10.1016/s0140-6736(07)61239-2

4. Saraceno B, van Ommeren M, Batniji R, Cohen A, Gureje O, Mahoney J, etal.Barriers to improvement of mental health services in low-income and middle-income countries. Lancet 2007; 370: 1164-74. doi: 10.1016/s0140-6736(07)61263-x

5. World Health Organization (WHO). Comprehensive Mental Health Action Plan 2013-2020. Sixty-Sixth World Health Assembly. Resolution WHA66/8. World Health Organization; 2013.

6. Faydi E, Funk M, Kleintjes S, Ofori-Atta A, Ssebunnya J, Mwanza $\mathrm{J}$, et al. An assessment of mental health policy in Ghana, South Africa, Uganda and Zambia. Health Res Policy Syst 2011; 9: 17. 10.1186/1478-4505-9-17

7. World Health Organization (WHO). Mental health policy, plans and programmes: WHO mental health policy and service guidance package - module 1 (update 2). Geneva: WHO; 2005.

8. Flisher AJ, Lund C, Funk M, Banda M, Bhana A, Doku V, Green A. Mental health policy development and implementation in four African countries. J Health Psychol 2007; 12: 505-16. doi: 10.1177/1359105307076237

9. World Health Organization Eastern Mediterranean Research Organization (WHO EMRO). Summary report of the intercountry meeting to scale-up action on mental health in the Eastern Mediterranean Region, Cairo, Egypt 15-17 September 2014.

10. Ssebunnya J, Kigozi F, Ndyanabangi S. Developing a National Mental Health Policy: A Case Study from Uganda. PLoS Med2012; 9: e1001319. doi: 10.1371/journal.pmed.1001319

11. Mackenzie J. Global mental health from a policy perspective: a context analysis. London: Overseas Development Institute; 2014.

12. Shiffman J, Okonofua FE. The state of political priority for safe motherhood in Nigeria. BJOG 2007; 114: 127-33. doi: 10.1111/j.1471-0528.2006.01184.x

13. The Mental Health Innovation Network (MHIN). Knowledge Exchange Strategy 2014-2017 [internet]. 2014 [cited 20 January 2015]. Available from: http://mhinnovation.net/resources/mhinknowledge-exchange-strategy-2014-2017\#.VMIpIEesWAU

14. De Silva M, Roland J; on behalf of the All Party Parliamentary Groups on Global Health and Mental Health. Mental Health for Sustainable Development. MHIN; 2014. 